\title{
Ursolic acid suppresses growth and adrenocorticotrophic hormone secretion in AtT20 cells as a potential agent targeting adrenocorticotrophic hormone-producing pituitary adenoma
}

\author{
YING-YING GONG ${ }^{1}$, YUAN-YUAN LIU ${ }^{1}$, SHUANG YU ${ }^{1}$, XIAO-NAN ZHU ${ }^{2}$, \\ XIAO-PEI CAO ${ }^{1}$ and HAI-PENG XIAO ${ }^{1}$ \\ ${ }^{1}$ Department of Endocrinology, The First Affiliated Hospital of Sun Yat-Sen University; \\ ${ }^{2}$ Department of Pharmacology, Zhong-Shan School of Medicine, Sun Yat-Sen University, \\ Guangzhou, Guangdong 510080, P.R. China
}

Received July 21, 2013; Accepted February 26, 2014

DOI: $10.3892 / \mathrm{mmr} .2014 .2078$

\begin{abstract}
Adrenocorticotrophic hormone (ACTH)-producing pituitary adenoma leads to excess ACTH secretion, which is associated with significant mortality and impaired quality of life. Thus far, the first line therapy is the transphenoidal microsurgery. Considering the high recurrence rate and complications of surgery, novel agents, which directly target on pituitary ACTH-producing adenoma and suppress ACTH secretion are urgently required. In the present study, the effect of ursolic acid (UA) as a candidate agent targeting ACTH-producing AtT20 cells was investigated. It was demonstrated that UA inhibited the viability and induced apoptosis of AtT20 cells and decreased ACTH secretion. The process of apoptosis involved a decrease of the $\mathrm{B}$ cell lymphoma 2 ( $\mathrm{Bcl}-2) / \mathrm{Bcl} 2$-associated $\mathrm{X}$ protein ratio followed by a release of mitochondrial cytochrome $c$ into the cytosol with subsequent activation of caspase- $9,-3 / 7$ and -8 . The potential signaling pathway involved the activation of c-Jun N-terminal kinase (JNK) but not extracellular signal-regulated protein kinases1/2 and p38 mitogen-activated protein kinase. The JNK pathway participated in UA-induced mitochondrial apoptotic signaling transduction via increasing the phosphorylation and degradation of Bcl-2, which may be partly attenuated by the JNK inhibitor SP600125. In conclusion, the present study indicates that UA may be a promising candidate agent for the management of ACTH-producing pituitary adenoma.
\end{abstract}

Correspondence to: Professor Hai-Peng Xiao, Department of Endocrinology, The First Affiliated Hospital of Sun Yat-Sen University, 58 Zhongshan Road 2, Guangzhou, Guangdong 510080, P.R. China

E-mail: xiaohp@mail.sysu.edu.cn

Key words: ursolic acid, apoptosis, adrenocorticotrophic hormone-producing pituitary adenoma, c-Jun N-terminal kinase, phosphorylated B-cell lymphoma 2

\section{Introduction}

Adrenocorticotrophic hormone (ACTH)-producing adenoma, accounting for $10-15 \%$ of pituitary tumors, result in Cushing's disease due to excessive secretion of ACTH and, consecutively, cortisol (1). Cushing's disease possesses a high morbidity and mortality rate if managed inadequately (1-3). Pituitary surgery, particularly transphenoidal microsurgery, remains the first choice amongst therapies. However, complication of hypopituitarism occurs in $\sim 80 \%$ of all cases (4) and the risk of recurrence reaches $20-25 \%$ at 10 years following surgery (5). Radiation therapy, which is often used in cases of persistence or recurrence, is limited by the risk of necrosis in the temporal lobe of the brain and high occurrence of long-term hypopituitarism (6). Medical therapy with classic adrenal-directed drugs (steroidogenesis inhibitors) may be highly effective but with severe side-effects, and these agents cannot inhibit underlying tumors or restore normal secretory dynamics (7-9). Pituitary-directed drugs, including somatostatin analogs and dopamine agonists, demonstrate certain effects on ACTH secretion in Cushing's disease; however, further long-term trials are required to determine the safety and efficacy (10-12). Other agents, such as peroxisome proliferator-activated receptor $\gamma$ (PPAR- $\gamma$ ) agonists for the treatment of Cushing's disease, are experimental and not currently available in human clinical trials $(13,14)$. As noted, the drugs that directly target the pituitary tumor growth and ACTH secretion are urgently required and would be an attractive option in the medical management of ACTH-producing pituitary adenoma.

Ursolic acid (UA) is a triterpenoid compound widely distributed in food, medicinal herbs and other plants (15). UA has various pharmacological properties, including anti-oxidant, anti-inflammatory and anti-hyperlipidemic activities $(16,17)$. In addition, UA has clinical applications for treating tumor patients as a promising antitumor agent (18). It has been proven that UA inhibited tumorigenesis and progression in a broad spectrum of tumors, including hepatocellular carcinoma, melanoma, and prostate, colorectal, breast and bladder cancer (19-26). The reported molecular 
mechanisms involved in UA-induced apoptosis include inhibition of nuclear factor $\mathrm{k}$-light-chain-enhancer of activated $\mathrm{B}$ cells $(\mathrm{NF}-\mathrm{\kappa B})$ activity and protein tyrosine kinase $(27,28)$. However, there is no report in the literature on the effect of UA on ACTH-producing pituitary adenoma and its potential molecular mechanisms.

In the present study, the effect of UA on apoptosis and ACTH secretion in AtT20 cells was investigated. The potential underlying molecular mechanisms of action, including endogenous, exogenous signaling pathways and the JNK pathway, were also explored.

\section{Materials and methods}

Cell culture and chemicals. AtT20 cells (mouse corticotroph tumor cell line) were provided by Shanghai Institute of Materia Medica (Shanghai, China) (29). AtT20 cells were cultured in RPMI-1640 medium supplemented with $10 \%$ fetal bovine serum (FBS) and 1\% penicillin-streptomycin (Invitrogen Life Technologies, Grand Island, NY, USA) at $37^{\circ} \mathrm{C}$ in $5 \% \mathrm{CO}_{2}$. UA purchased from Sigma-Aldrich (St. Louis, MO, USA) was dissolved in dimethyl sulfoxide (DMSO; Sigma-Aldrich) as a $100 \mathrm{mM}$ stock solution and stored at $-20^{\circ} \mathrm{C}$. The cells were pretreated with JNK inhibitor SP600125 (Calbiochem, La Jolla, CA, USA), which was dissolved in DMSO $1 \mathrm{~h}$ prior to UA treatment.

\section{2-(2-Methoxy-4-nitrophenyl)-3-(4-nitrophenyl)-5-(2,4-disulfo} phenyl)-2H-tetrazolium [Cell Counting kit-8 (CCK-8)] assay. Subsequent to being harvested and centrifuged, the AtT20 cells were resuspended in RPMI-1640 medium with 1\% FBS and seeded in a 96-well plate at a density of $1 \times 10^{5} /$ well. The cells were treated with different concentrations of UA for 24, 48 and $72 \mathrm{~h}$, respectively, while only DMSO was added to the control wells. A total of $10 \mu \mathrm{l} \mathrm{CCK-8} \mathrm{(Dojindo,} \mathrm{Kumamoto,} \mathrm{Japan)} \mathrm{was}$ added to each well and the microplate was incubated for $6 \mathrm{~h}$ at $37^{\circ} \mathrm{C}$ with $5 \% \mathrm{CO}_{2}$. The optical density (OD) at $450 \mathrm{~nm}$ was read with a 96-well plate reader (Bio-Rad, Reinach, Switzerland). Experiments were conducted with five replicates.

Annexin V-fluorescein/propidium iodide (PI) flow cytometric analysis. AtT20 cells were collected, centrifuged and washed with phosphate-buffered saline following treatment with the indicated amount of UA for $24 \mathrm{~h}$ with or without pretreatment of SP600125 (JNK inhibitor; Sigma-Aldrich) for $1 \mathrm{~h}$ followed by staining with Annexin V-fluorscein (FLOUS) Staining kit and PI (Roche Diagnostics, Mannheim, Germany) for $15 \mathrm{~min}$ at room temperature. For each example, 20,000 cells were analyzed on a flow cytometer (FACStar, BD Biosciences, Franklin Lanes, NJ, USA).

Assessment of caspase-3/7, -8 and -9 activity. The caspase-3/7, -8 and -9 activities were measured by Caspase-Glo luminescent-based assays (Caspase-Glo kit; Promega, Madison, WI, USA). The cells were seeded with a total of $10^{5}$ cells/well in a 96-well plate. Following treatment with UA (at various concentrations) in combination with SP600125 for $24 \mathrm{~h}, 100 \mu \mathrm{l}$ of the Caspase-Glo-3/7, -8, -9 reagents were added to each well. The mixtures were incubated for $1 \mathrm{~h}$ and then transferred to a fluorescence microtiter plate. Quantification of luminescence

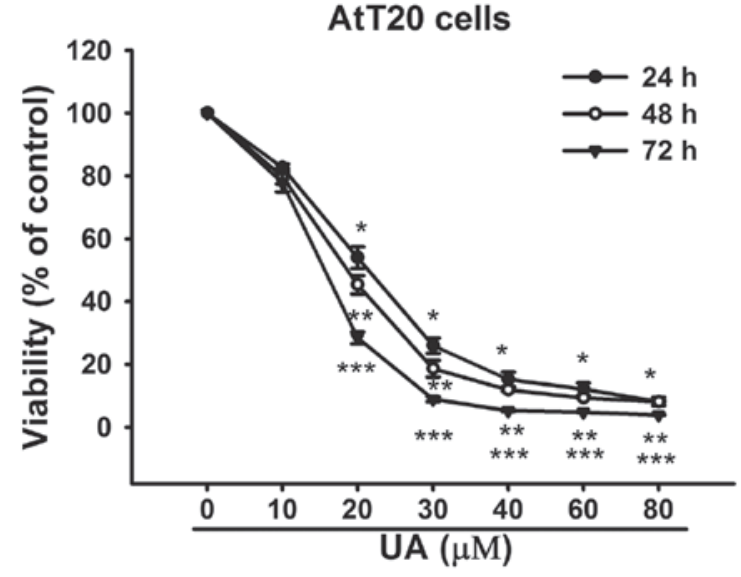

Figure 1. UA inhibits viability of AtT20 cells. AtT20 cells were treated with the indicated concentrations of UA and cell viability was determined by the CCK-8 assay after 24,48 and $72 \mathrm{~h}$, respectively. The data were calculated as $\%$ of vehicle control and expressed as the mean \pm standard deviation of three separate experiments, each performed with five replicates. ${ }^{*} \mathrm{P}<0.05 \mathrm{vs}$. control 24 h group, ${ }^{* *} \mathrm{P}<0.05$ vs. control 48 h group, ${ }^{* * * *} \mathrm{P}<0.05$ vs. control 72 h group. CCK-8, cell counting kit-8; UA, ursolic acid.

was measured by a luminometer. To normalize the fluorescence intensity, the optical density values of the CCK- 8 assay were measured by a Infinite F500 microplate reader (Tecan, Männedorf, Switzerland) as the viable cell number.

quantitative polymerase chain reaction ( $q P C R$ ) analysis. The total RNA was extracted from cells with indicated treatments. A volume of $5 \mu \mathrm{l}$ cDNA (ReverTra Ace qPCR RT kit, Toyobo, Osaka, Japan) was amplified in a final volume of $0.4 \mu \mathrm{M}$ of each primer and $25 \mu 1$ SYBR Green Real-time PCR Master Mix (Toyobo) in a final volume of $50 \mu 1$ for 40 cycles (ABI Prism 7500 Sequence Detection system; Applied Biosystems, Carlsbad, CA, USA). The primers were as follows: Pro-opio melanocortin (POMC) forward, 5'-AACCTGCTGGCTTGCATCCG-3' and reverse, 5'-GGGC TGTTCATCTCCGTTGCCT-3'. $\beta$-actin, forward, 5'-TGGAATC CTGTGGCATCCATGAAAC-3' and reverse, 5'-TAAAACGCAG CTCAGTAACAGTCC-3'.

Assessment of ACTH by ELISA. The AtT20 cells were harvested, seeded in 24-well plates at a density of $2 \times 10^{6} / 500 \mu 1 /$ well and then added to different concentrations of UA for $48 \mathrm{~h}$. The cell culture supernatants were collected and centrifuged. ACTH was assayed using a mouse ACTH ELISA kit (Uscn Life Science Inc., Wuhan, China) following the manufacturer's instructions.

Western blot analysis. Protein samples were extracted in radio-immunoprecipitation assay lysis buffer containing protease inhibitors and phosphatase inhibitors (Thermo Fisher Scientific, Waltham, MA, USA). Mitochondria were extracted using a mitochondrial isolation kit (Thermo Fisher Scientific). Following incubation for 10-30 min at $4^{\circ} \mathrm{C}$, the supernatant was collected by centrifugation $(10,000 \mathrm{x} \mathrm{g}$, $\left.15 \mathrm{~min}, 4^{\circ} \mathrm{C}\right)$. The cell lysates $(20-40 \mu \mathrm{g})$ which were determined using the bicinchoninic acid protein assay kit (Pierce Chemical Co., Rockford, IL, USA) proteins were separated by $12 \%$ SDS-PAGE and electrophoretically transferred onto polyvinylidene difluoride membranes (Millipore, Billerica, MA, 
A

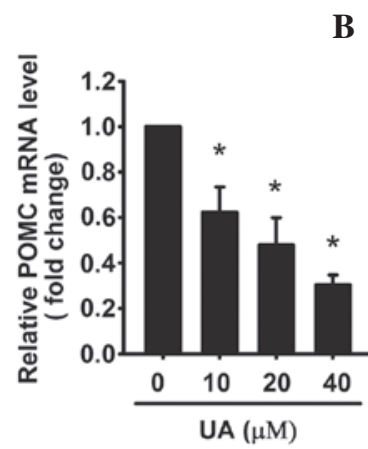

B

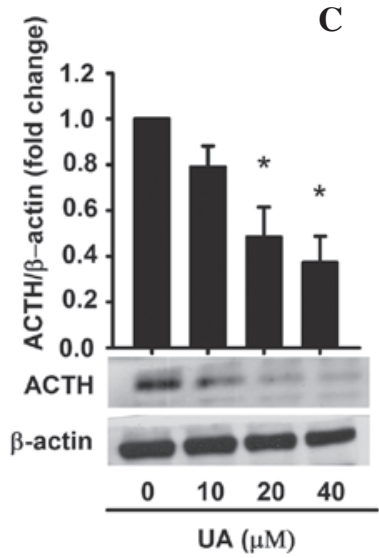

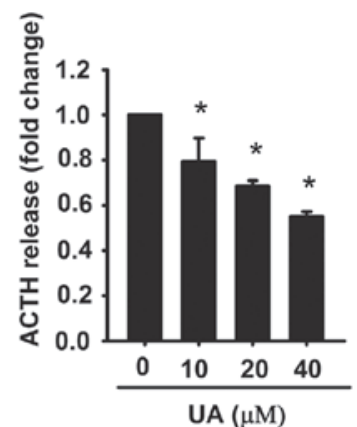

Figure 2. UA decreased mRNA levels intracellular protein levels, and the secretion of ACTH. The AtT20 cells were treated with the indicated concentrations of UA for $24 \mathrm{~h}$, then (A) the expression of POMC mRNA (precursor of ACTH) was detected by quantitative polymerase chain reaction; (B) the intracellular protein levels of ACTH were detected by western blot analysis and (C) and an equal amount of culture supernatant was used to detect ACTH secretion from AtT20 cells. The data are presented as the fold-change compared with the control. Each value is the mean \pm standard deviation of three separate experiments. ${ }^{*} \mathrm{P}<0.05$ vs. control. POMC, pro-opio melanocortin; ACTH, adrenocorticotrophic hormone; UA, ursolic acid.

USA). Membranes were washed twice in Tris-buffered saline and Tween 20 (TBST) and incubated with blocking buffer (5\% skimmed milk in TBST) for $60 \mathrm{~min}$ at room temperature. Next, the membranes were washed three times and incubated overnight at $4^{\circ} \mathrm{C}$ with primary antibodies. The membranes were incubated with secondary antibodies (1:1,000; Cell Signaling Technology, Inc., Danvers, MA, USA) for $1 \mathrm{~h}$ at room temperature. Following washing three times, the antibodies bound to the proteins were detected using enhanced chemiluminescence (Invitrogen Life Technologies). The following primary antibodies were used: anti-ACTH antibody (Ab) $(1: 1,000$; Abcam); anti-phospho-p42/44 mitogen-activated protein kinase (MAPK) (Thr202/Tyr204) Ab, anti-p42/44MAPK Ab, anti-phospho-p38 (Thr180/Tyr182) Ab, anti-p38 Ab, anti-phospho-JNK (Thr183/Tyr185) Ab, anti-JNK Ab, anti-B cell lymphoma 2 (Bcl-2) Ab, anti-Bcl-2-associated X (Bax) $\mathrm{Ab}$, anti- $\beta$-actin Ab (all 1:1,000; Cell Signaling Technology, Inc.); anti-phospho-Bcl-2 (Ser70) Ab (1:1,000; Sigma-Aldrich); anti-cytochrome $c$ Ab (1:1,000; Santa Cruz Biotechnology, Inc, Santa Cruz, CA, USA). First, the phospho-specific MAPK were detected. Next, the membranes were stripped and re-probed with anti-JNK, anti-extracellular signal-regulated protein kinases (ERK) and anti-p38 antibodies. The densities of phosphorylated MAPK bands were normalized to that of the total MAPK bands. The expression levels of p-Bcl-2 and total $\mathrm{Bcl}-2$ were assessed in the same manner. The secondary antibodies were Goat anti-rabbit IgG.

Statistical analysis. The results were expressed as the mean \pm standard deviation of three separate experiments. Data were analyzed by one-way analysis of variance followed by Fisher's least significant difference test or by Kruskal-Wallits test using SPSS 11 version software. $\mathrm{P}<0.05$ was used to indicate a statistically significant difference.

\section{Results}

UA reduces viability and induces apoptosis of AtT20 cells. In order to investigate the effect of UA on cytotoxicity, the AtT20 cells were exposed to UA (10-100 $\mu \mathrm{M})$ for 24,48 and $72 \mathrm{~h}$, respectively. The CCK-8 results revealed that UA inhibited the viability of AtT20 cells in a dose- and time-dependent manner (Fig. 1). The $\mathrm{IC}_{50}$ value for $24 \mathrm{~h}$ was $20.02 \mu \mathrm{M}$. Considering the $\mathrm{IC}_{50}$ for $24 \mathrm{~h}$, the concentration of $20 \mu \mathrm{M}$ was used for further analysis.

The apoptosis of AtT20 cells induced by UA was further evaluated. AtT 20 cells were treated with $\operatorname{UA}(10,20$ and $40 \mu \mathrm{M})$ for $24 \mathrm{~h}$, respectively. Afterwards, Annexin V-FLOUS/PI staining was performed and assessed by flow cytometry. The percentage of apoptotic cells, including early apoptotic cells (Annexin $\mathrm{V}$-FLOUS ${ }^{+} / \mathrm{PI}^{-}$) and late apoptotic cells (Annexin $\mathrm{V}-\mathrm{FLOUS}^{+} / \mathrm{PI}^{+}$) was significantly increased in a dose-dependent manner (Fig. 3A). In addition, the activity of caspase-3/7, which is the executioner of apoptosis, was examined. Caspase-3/7 activity was gradually enhanced from 2.8- to 13.7-fold as the dose of UA increased (Fig. 3D). These results confirm that cell viability loss induced by UA in AtT20 cells is facilitated by the induction of apoptosis.

UA decreases ACTH production and secretion in AtT20 cells. Next, it was investigated whether UA-induced apoptosis was accompanied with a decreased ACTH secretion. It has been reported that $\mathrm{ACTH}$ is derived from the POMC precursor peptide (30). In order to examine the effect on the intracellular levels of ACTH, UA (10, 20 and $40 \mu \mathrm{M})$ was added to AtT20 cells for $48 \mathrm{~h}$, respectively, and the extracted total RNA was analyzed by qPCR. The results demonstrated that UA inhibited POMC mRNA (precursor of ACTH) in a dose-dependent manner. $40 \mu \mathrm{M}$ UA achieved a maximum effect of inhibition ( $\sim 0.3$-fold compared with the control) (Fig. 2A). Western blot analysis also proved that UA decreased ACTH synthesis in a dose-dependent manner (Fig. 2B).

For determining the levels of ACTH, the conditioned medium was harvested and analyzed by ELISA. The data demonstrated that ACTH was reduced by UA in a dose-dependent manner. Compared with the control, ACTH levels were 0.62-, 0.48-, 0.3-fold, respectively (Fig. 2C). These results indicated that UA decreased the expression of POMC mRNA as well as ACTH synthesis and secretion in AtT20 cells in a dose-dependent manner. 
A
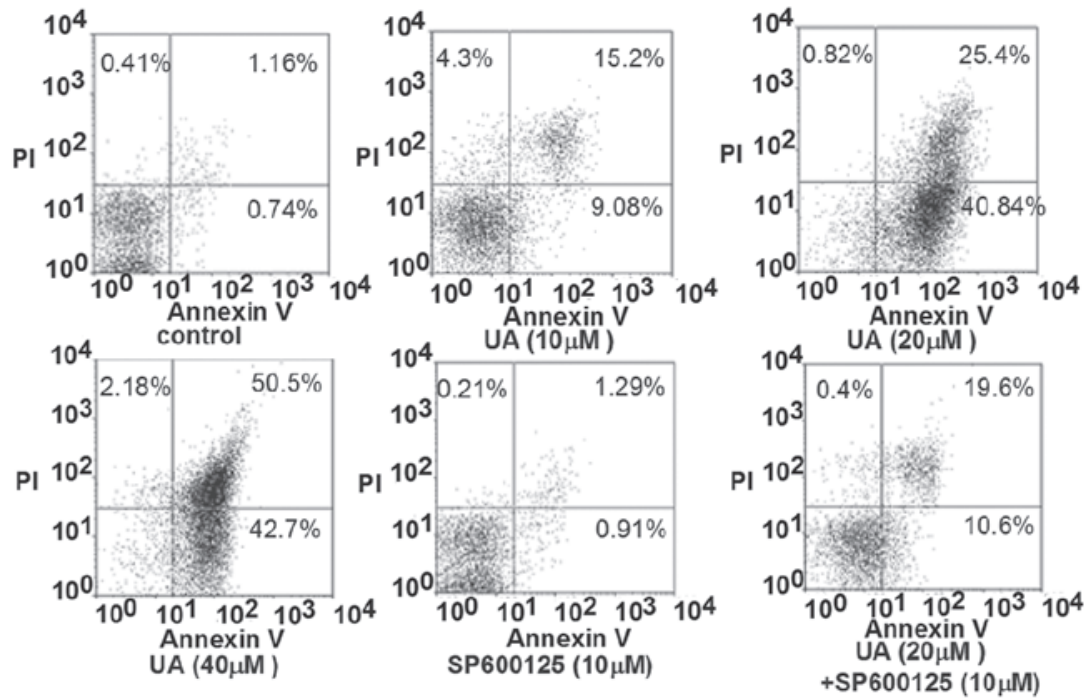

B

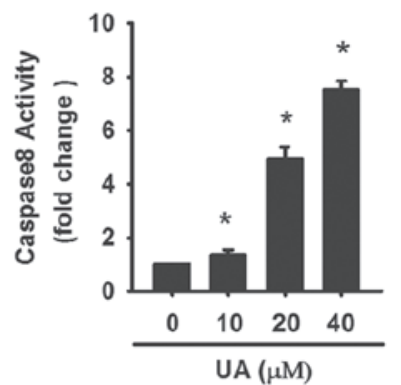

$\mathbf{E}$

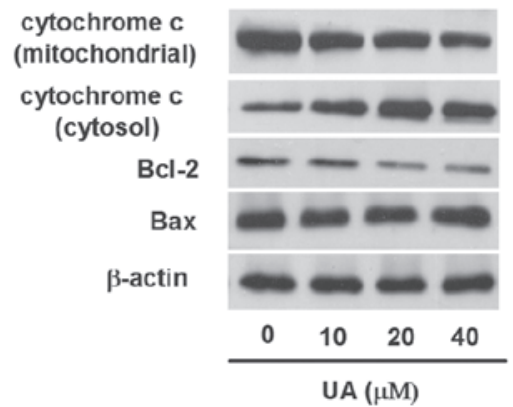

C

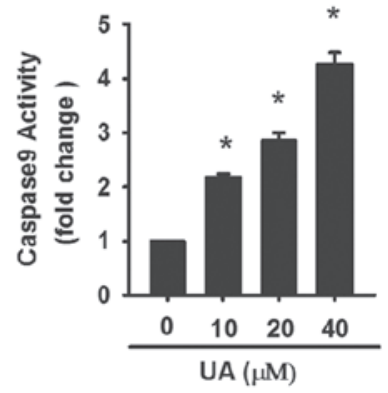

$\mathbf{F}$

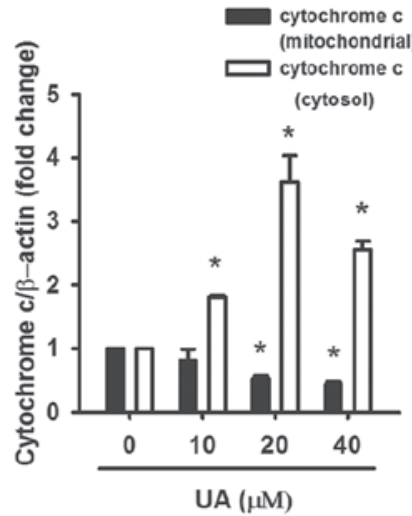

D
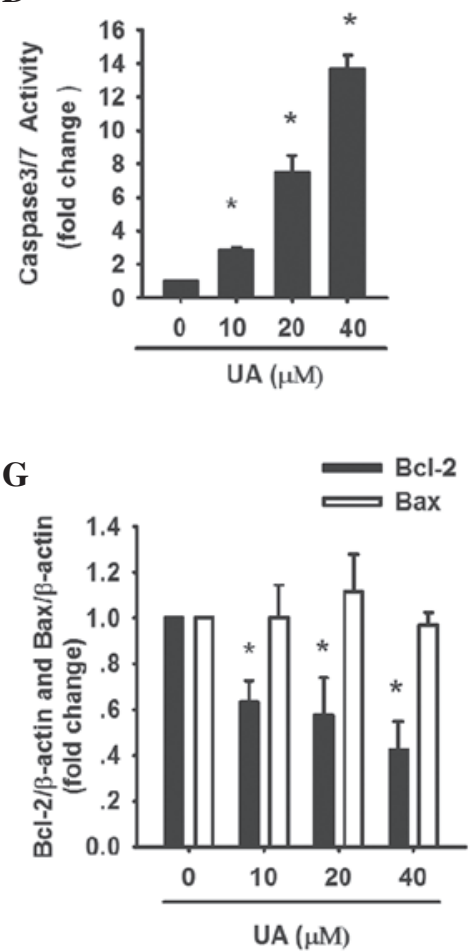

Figure 3. UA induces apoptosis in AtT20 cells. AtT20 cells were treated with 10, 20 and $40 \mu \mathrm{M}$ UA for 24 h in the presence or absence of $10 \mu \mathrm{M}$ SP600125 for 1 h. (A) Representative dot plots and percentages of AtT20 cells stained with Annexin V-fluorescein and PI. (B) Effect of UA on the caspase-8, (C) caspase-9 and (D) caspase-3/7 activities by caspase-Glo luminescent-based assays. (E) Effect of UA on protein levels of cytochrome $c$ in mitochondria and cytosol as well as Bcl-2 and Bax levels by western blot analysis. (F) Respective fold-change in protein levels of cytochrome $c$ in mitochondria and cytosol. (G) Respective fold-change in protein levels of Bcl-2 and Bax. Each value was the mean \pm standard deviation of three separate experiments. * $\mathrm{P}<0.05 \mathrm{vs}$. control; ${ }^{\# \#} \mathrm{P}<0.05$ vs. UA (20 $\mu \mathrm{M})$. Bcl-2, B cell lymphoma 2; Bax, Bcl2-associated X protein; UA, ursolic acid; PI, propidium iodide.

Endogenous and exogenous signaling pathways are involved in UA-induced apoptosis. Apoptosis is mediated by endogenous and exogenous signal transduction. To address whether the two pathways were involved in UA-induced apoptosis, caspase-8, -9, cytochrome $c, \mathrm{Bcl}-2$ and Bax were assayed. AtT20 cells were treated with UA $(10,20$ and $40 \mu \mathrm{M})$ for 24 h. Caspase- 8 activity increased by 1.35-, 4.93-, 7.5-fold, respectively, as compared with the control (Fig. 3B). The levels of Bcl-2 and cytochrome $c$ in mitochondria were reduced while cytochrome $c$ in cytosol was increased along with increasing dose of UA (Fig. 3E and F). The protein levels of Bax were normal while the ratio of $\mathrm{Bcl}-2 / \mathrm{Bax}$ decreased (Fig. 3E and G). Caspase-9 was also significantly increased by 4.27-fold subsequent to treatment with $40 \mu \mathrm{M}$ UA (Fig. 3C). The results revealed that endogenous and exogenous signaling pathways contributed to UA-induced apoptosis. 
A

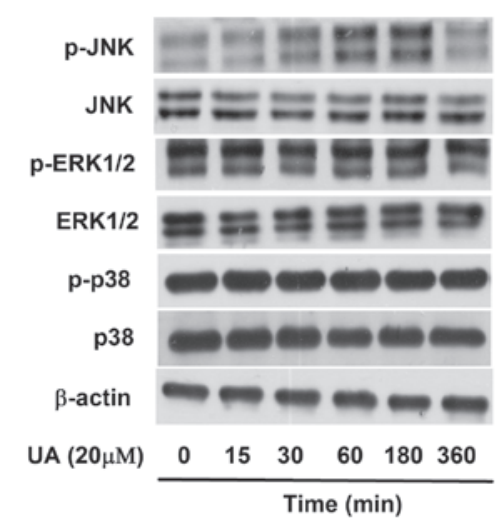

C

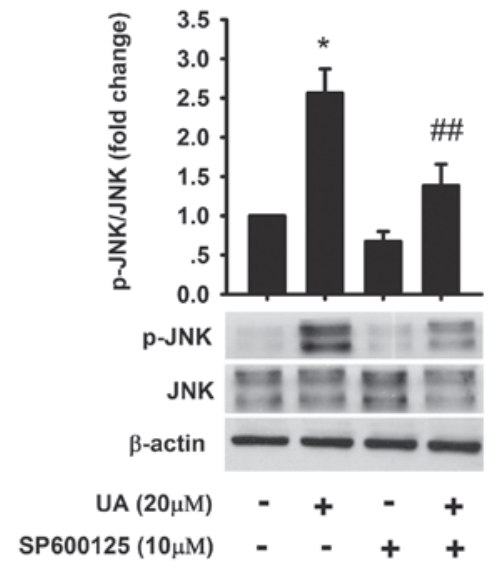

B
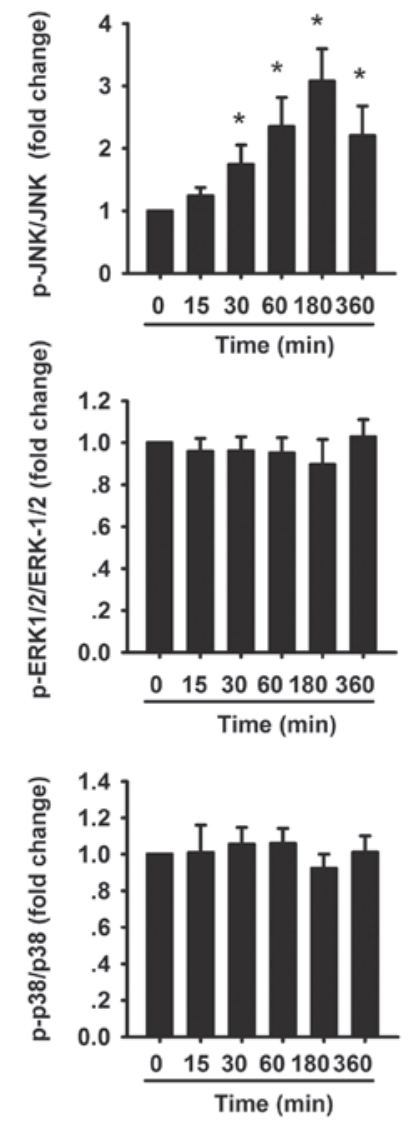

Figure 4. Effect of UA on the activation of JNK, ERK1/2 and p38 in AtT20 cells. (A,B) AtT20 cells were incubated with 20 $\mu$ M UA for 15, $30,60,180$ and 360 min. Protein levels of JNK, p-JNK, ERK1/2, p-ERK1/2, p38 and p-p38 were detected by western blot analysis. (C) AtT20 cells were treated with $10 \mu \mathrm{M}$ UA or SP600125 for $1 \mathrm{~h}$ prior to $20 \mu \mathrm{M}$ UA treatment. Next, the effect of JNK inhibitor SP600125 on JNK and p-JNK expression was detected by western blot analysis. The data are presented as the fold-change of the control. Each value was the mean \pm standard deviation of three separate experiments. ${ }^{*} \mathrm{P}<0.05$ vs. control; ${ }^{\#} \mathrm{P}<0.05$ vs. UA $(20 \mu \mathrm{M})$. p-JNK, phosphorylated c-Jun N-terminal kinase; p-ERK, phosphorylated extracellular signal-regulated protein kinase; UA, ursolic acid.
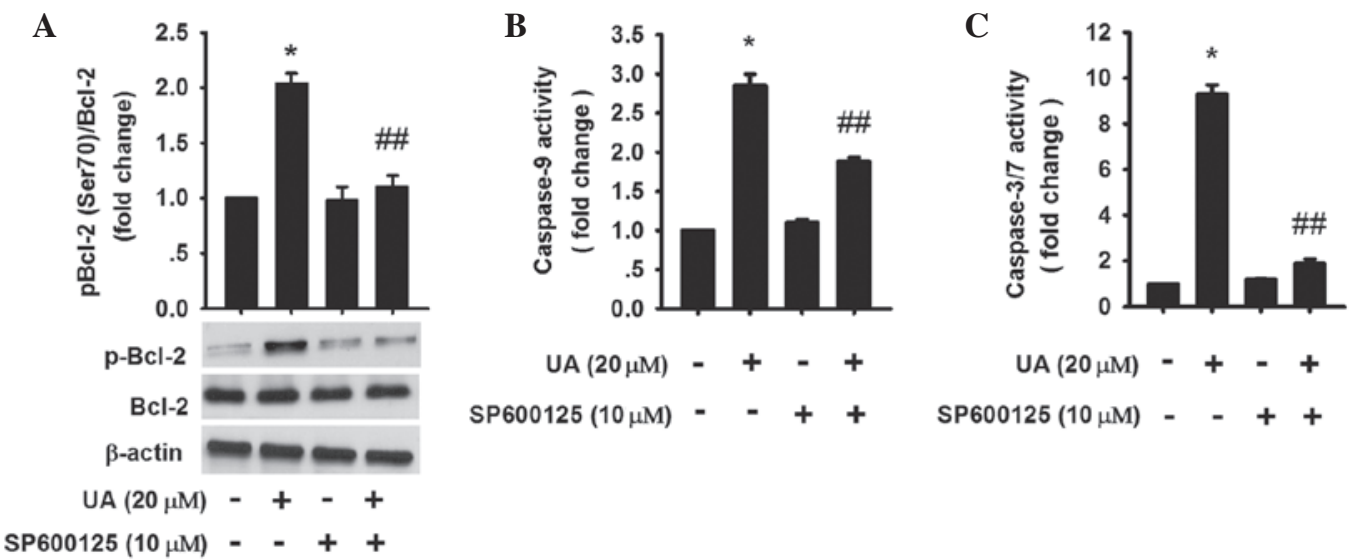

Figure 5. Involvement of the JNK pathway in UA-induced apoptosis in AtT20 cells. (A) Expression levels of p-Bcl-2 and Bcl-2 were detected by western blot analysis. AtT20 cells were incubated with $20 \mu \mathrm{M}$ UA for $3 \mathrm{~h}$ in the presence or absence of SP600125 for $1 \mathrm{~h}$. The effect of $20 \mu \mathrm{M}$ UA was assessed on (B) caspase- 9 and (C) caspase-3/7 activity for $24 \mathrm{~h}$ with or without pretreatment with $10 \mu \mathrm{M} \mathrm{SP} 600125$ for $1 \mathrm{~h}$. Data are presented as fold-change of the control. Each value was the mean \pm standard deviation of three separate experiments. ${ }^{*} \mathrm{P}<0.05$ vs. control; ${ }^{\# \prime} \mathrm{P}<0.05$ vs. UA $(20 \mu \mathrm{M})$. JNK, c-Jun N-terminal kinase; Bcl2-associated X protein; UA, ursolic acid.

The JNK pathway is involved in UA-induced apoptosis. Cell proliferation and apoptosis, and the MAPK family are closely associated (31). UA has been shown to activate the MAPK signaling pathway in various cells $(23,32)$. To further investigate the exact molecular mechanism of UA-induced apoptosis and identify whether the MAPK signaling pathway was also activated by UA in AtT20 cells, phosphorylation of JNK, ERK-1/2 and p38 was detected. The results revealed that $20 \mu \mathrm{M}$ UA upregulated the expression of phosphorylation of JNK, which reached a maximum from 1 to $3 \mathrm{~h}$, but 
had no effect on the phosphorylation of ERK-1/2 and p38 (Fig. 4A and B). To further confirm whether JNK activation mediated UA-induced apoptosis, AtT20 cells were pretreated with $10 \mu \mathrm{M}$ SP600125 (JNK inhibitor) for $1 \mathrm{~h}$ followed by $20 \mu \mathrm{M}$ UA for $24 \mathrm{~h}$ (Fig. 4C). The percentages of early and late apoptotic cells were 19.6 and $10.6 \%$ respectively, which were significantly less compared with that of cells in the group treated with $20 \mu \mathrm{M}$ UA only (Fig. 3A).

It has been reported that JNK activation inactivated $\mathrm{Bcl}-2$ by increasing phosphorylation of Bcl-2 $(33,34)$. The results showed that $20 \mu \mathrm{M}$ UA treatment for $3 \mathrm{~h}$ increased phosphorylated-Bcl-2 (Ser 70), while inhibition of JNK activation by SP600125 could downregulate the phosphorylation (Fig. 5A), demonstrating that JNK activation was involved in the upregulation of UA-induced $\mathrm{Bcl}-2$ phosphorylation and resulting apoptosis.

To evaluate whether the activation of caspases was affected by Bcl-2, caspase- 9 and $-3 / 7$ were assessed. The results revealed that caspase-3/7 and -9 activation were partly blocked in the UA group in the presence of SP600125 (Fig. 5A-C). Thus, the data indicated that JNK activation was involved in the endogenous signaling pathway in UA-induced apoptosis by increasing phosphorylated Bcl-2.

\section{Discussion}

Pituitary adenoma accounts for a significant proportion of primary intracerebral tumors. Although ACTH-producing pituitary adenoma is benign, it does cause detrimental effects due to excess hormone secretion resulting in significant mortality and an impaired quality of life. Removing or shrinking the tumor and normalization of ACTH excess are the crucial goals of treatment. Thus far, no reliable medical therapies exist to directly target the pituitary tumor growth and ACTH secretion. In the present study, it was demonstrated that UA could function as a potential novel and potent therapeutic agent targeting directly on ACTH-producing pituitary adenomas.

The present study revealed that UA was able to reduce the viability and induce apoptosis of AtT20 cells (mouse corticotroph tumor cell line) in a dose-dependent manner. The results were in consistency with other studies in various tumor types (21,35-39), which indicated that UA had a pro-apoptotic effect on ACTH-producing pituitary tumor cells.

Excess ACTH levels and hypercortisolemia may result in high co-morbidity and mortality. Normalization of hormone excess is the therapeutic goal of treatment. Numerous compounds, including dopamine agonists, thiazolidinediones and curcumin have been reported to inhibit ACTH synthesis and (or) secretion $(12,40,41)$. However, none of these agents has been proven to be effective in the management of ACTH-producing pituitary adenoma. In the present study, it was identified that UA decreased ACTH production and secretion in AtT20 cells in a dose-dependent manner, demonstrating the potential of UA to be a novel agent for the management of ACTH-producing pituitary adenoma.

Apoptosis has an essential role in tumorigenesis. Two major apoptotic pathways have been identified (42). One is the exogenous pathway, which involves the binding of a ligand to the death receptor and subsequent caspase- 8 activation (43). The other is the endogenous pathway, relying on the release of cytochrome $c$ from mitochondria to the cytosol which recruits the initiator pro-caspase-9, which yields activated caspase-9 and finally activates caspase-3 (44). The key components of the mechanism involved in mitochondria-dependent apoptosis are the Bcl-2 family of proteins, including pro-apoptotic Bax and anti-apoptotic Bcl-2 proteins (45). Bcl-2 proteins usually form heterodimers with Bax, resulting in the release of cytochrome $c$ from mitochondria to the cytosol and triggering the death program. In the present study, it was revealed that UA increased caspase- 9 and -8 activities, decreased the ratio of Bcl-2/Bax and promoted cytochrome $c$ release from mitochondria in a dose-dependent manner. The results indicated that exogenous and endogenous pathways were involved in UA-induced apoptosis in AtT20 cells.

To elucidate how UA triggered the apoptotic process, the exact molecular mechanisms were further investigated. It has been reported that the MAPK pathway had a vital role in UA-induced apoptosis in various tumor cells $(32,37,46)$. In the present study, UA was found to increase the phosphorylation of JNK, but not ERK1/2 or p38, in a time-dependent manner. Pretreatment with JNK inhibitor SP600125 blocked UA-induced cleavage of caspase-3 and -9. Previous studies have reported that the JNK pathway participated in UA-induced the apoptotic signaling pathway via controlling phosphorylation of Bcl-2 $(36,37,47)$. The phosphorylation of $\mathrm{Bcl}-2$ resulted in the degradation of $\mathrm{Bcl}-2$, which led to the release of $\mathrm{Bax}$ from the $\mathrm{Bcl}-2 / \mathrm{Bax}$ heterodimer and triggered apoptosis (48). The present study demonstrated that UA treatment for $24 \mathrm{~h}$ decreased the levels of Bcl-2 and additionally induced the phosphorylation of Bcl-2 in AtT20 cells. Furthermore, pretreatment with SP600125 was able to partly block UA-induced Bcl-2 phosphorylation (Ser70) and degradation. These findings revealed that UA-induced JNK activation may promote $\mathrm{Bcl}-2$ phosphorylation, degradation and finally induce apoptosis.

In conclusion, the present study demonstrated that UA inhibited viability, induced apoptosis and decreased ACTH production in AtT20 cells. The induction of apoptosis involved exogenous and endogenous pathways. Increased phosphorylation of Bcl-2 via JNK activation had a crucial role in UA-induced apoptosis in AtT20 cells. These findings indicate the potential of UA as a novel potential therapeutic agent targeting ACTH-producing pituitary adenoma. Further clinical studies are required to examine the efficacy and safety of UA.

\section{Acknowledgements}

The abstract of this study has been published in Endocr Rev Vol. 34, (03 Meeting Abstracts): Sun-188, 2013.

\section{References}

1. Boscaro M, Barzon L, Fallo F and Sonino N: Cushing's syndrome. Lancet 357: 783-791, 2001.

2. Arnaldi G, Angeli A, Atkinson AB, et al: Diagnosis and complications of Cushing's syndrome: a consensus statement. J Clin Endocrinol Metab 88: 5593-5602, 2003.

3. Witek P, Zielinski G, Szamotulska K, Witek J and Zgliczynski W: Complications of Cushing's disease - prospective evaluation and clinical characteristics. Do they affect the efficacy of surgical treatment? Endokrynol Pol 63: 277-285, 2012.

4. Swearingen B: Update on pituitary surgery. J Clin Endocrinol Metab 97: 1073-1081, 2012. 
5. Atkinson AB, Kennedy A, Wiggam MI, McCance DR and Sheridan B: Long-term remission rates after pituitary surgery for Cushing's disease: the need for long-term surveillance. Clin Endocrinol (Oxf) 63: 549-559, 2005

6. Heaney AP: Clinical review: pituitary carcinoma: difficult diagnosis and treatment. J Clin Endocrinol Metab 96: 3649-3660, 2011.

7. Beardwell CG, Adamson AR and Shalet SM: Prolonged remission in florid Cushing's syndrome following metyrapone treatment. Clin Endocrinol (Oxf) 14: 485-492, 1981.

8. Boscaro M, Sonino N, Rampazzo A and Mantero F: Response of pituitary-adrenal axis to corticotrophin releasing hormone in patients with Cushing's disease before and after ketoconazole treatment. Clin Endocrinol (Oxf) 27: 461-467, 1987.

9. Sonino N, Boscaro M and Fallo F: Pharmacologic management of Cushing syndrome: new targets for therapy. Treat Endocrinol 4 : 87-94, 2005.

10. Petersenn S, Schopohl J, Barkan A, et al: Pasireotide (SOM230) demonstrates efficacy and safety in patients with acromegaly: a randomized, multicenter, phase II trial. J Clin Endocrinol Metab 95: 2781-2789, 2010

11. Bode H, Seiz M, Lammert A, et al: SOM230 (pasireotide) and temozolomide achieve sustained control of tumour progression and ACTH secretion in pituitary carcinoma with widespread metastases. Exp Clin Endocrinol Diabetes 118: 760-763, 2010.

12. Pivonello R, Ferone D, de Herder WW, et al: Dopamine receptor expression and function in corticotroph pituitary tumors. J Clin Endocrinol Metab 89: 2452-2462, 2004

13. Mullan KR, Leslie H, McCance DR, Sheridan B and Atkinson AB: The PPAR-gamma activator rosiglitazone fails to lower plasma ACTH levels in patients with Nelson's syndrome. Clin Endocrinol (Oxf) 64: 519-522, 2006

14. Pecori Giraldi F, Scaroni C, Arvat E, et al: Effect of protracted treatment with rosiglitazone, a PPARgamma agonist, in patients with Cushing's disease. Clin Endocrinol (Oxf) 64: 219-224, 2006

15. Liu J: Pharmacology of oleanolic acid and ursolic acid. J Ethnopharmacol 49: 57-68, 1995.

16. Sultana N and Ata A: Oleanolic acid and related derivatives as medicinally important compounds. J Enzyme Inhib Med Chem 23: 739-756, 2008

17. Sultana N: Clinically useful anticancer, antitumor, and antiwrinkle agent, ursolic acid and related derivatives as medicinally important natural product. J Enzyme Inhib Med Chem 26 : 616-642, 2011

18. Liu J: Oleanolic acid and ursolic acid: research perspectives. J Ethnopharmacol 100: 92-94, 2005.

19. Kassi E, Papoutsi Z, Pratsinis H, Aligiannis N, Manoussakis M and Moutsatsou P: Ursolic acid, a naturally occurring triterpenoid, demonstrates anticancer activity on human prostate cancer cells. J Cancer Res Clin Oncol 133: 493-500, 2007.

20. Shih WL, Yu FL, Chang CD, Liao MH, Wu HY and Lin PY: Suppression of AMF/PGI-mediated tumorigenic activities by ursolic acid in cultured hepatoma cells and in a mouse model. Mol Carcinog 52: 800-812, 2013.

21. Shyu MH, Kao TC and Yen GC: Oleanolic acid and ursolic acid induce apoptosis in $\mathrm{HuH7}$ human hepatocellular carcinoma cells through a mitochondrial-dependent pathway and downregulation of XIAP. J Agric Food Chem 58: 6110-6118, 2010.

22. Prasad S, Yadav VR, Sung B, et al: Ursolic acid inhibits growth and metastasis of human colorectal cancer in an orthotopic nude mouse model by targeting multiple cell signaling pathways: Chemosensitization with capecitabine. Clin Cancer Res 18 4942-4953, 2012

23. Shan JZ, Xuan YY, Zheng S, Dong Q and Zhang SZ: Ursolic acid inhibits proliferation and induces apoptosis of HT-29 colon cancer cells by inhibiting the EGFR/MAPK pathway. J Zhejiang Univ Sci B 10: 668-674, 2009.

24. Harmand PO, Duval R, Delage C and Simon A: Ursolic acid induces apoptosis through mitochondrial intrinsic pathway and caspase-3 activation in M4Beu melanoma cells. Int J Cancer 114 $1-11,2005$.

25. De Angel RE, Smith SM, Glickman RD, Perkins SN and Hursting SD: Antitumor effects of ursolic acid in a mouse model of postmenopausal breast cancer. Nutr Cancer 62: 1074-1086, 2010 .
26. Zheng QY, Jin FS, Yao C, Zhang T, Zhang GH and Ai X: Ursolic acid-induced AMP-activated protein kinase (AMPK) activation contributes to growth inhibition and apoptosis in human bladder cancer T24 cells. Biochem Biophys Res Commun 419: 741-747, 2012.

27. Zhu W, Ou Y, Li Y, et al: A small-molecule triptolide suppresses angiogenesis and invasion of human anaplastic thyroid carcinoma cells via down-regulation of the nuclear factor-kappa B pathway. Mol Pharmacol 75: 812-819, 2009.

28. Hollosy F, Meszaros G, Bokonyi G, et al: Cytostatic, cytotoxic and protein tyrosine kinase inhibitory activity of ursolic acid in A431 human tumor cells. Anticancer Res 20: 4563-4570, 2000.

29. Gamby C, Waage MC, Allen RG and Baizer L: Growth-associated protein-43 (GAP-43) facilitates peptide hormone secretion in mouse anterior pituitary AtT-20 cells. J Biol Chem 271: 10023-10028, 1996.

30. White A and Gibson S: ACTH precursors: biological significance and clinical relevance. Clin Endocrinol (Oxf) 48: 251-255, 1998.

31. Chang L and Karin M: Mammalian MAP kinase signalling cascades. Nature 410: 37-40, 2001

32. Liu XS and Jiang J: Induction of apoptosis and regulation of the MAPK pathway by ursolic acid in human leukemia K562 cells. Planta Med 73: 1192-1194, 2007.

33. Ruvolo PP, Deng X and May WS: Phosphorylation of Bcl2 and regulation of apoptosis. Leukemia 15: 515-522, 2001.

34. Miyoshi N, Uchida K, Osawa T and Nakamura Y: A link between benzyl isothiocyanate-induced cell cycle arrest and apoptosis: involvement of mitogen-activated protein kinases in the Bcl-2 phosphorylation. Cancer Res 64: 2134-2142, 2004.

35. Bonaccorsi I, Altieri F, Sciamanna I, et al: Endogenous reverse transcriptase as a mediator of ursolic acid's anti-proliferative and differentiating effects in human cancer cell lines. Cancer Lett 263: 130-139, 2008

36. Zhang YX, Kong CZ, Wang HQ, Wang LH, Xu CL and Sun YH: Phosphorylation of $\mathrm{Bcl}-2$ and activation of caspase-3 via the c-Jun N-terminal kinase pathway in ursolic acid-induced DU145 cells apoptosis. Biochimie 91: 1173-1179, 2009.

37. Zhang Y, Kong C, Zeng Y, et al: Ursolic acid induces PC-3 cell apoptosis via activation of JNK and inhibition of Akt pathways in vitro. Mol Carcinog 49: 374-385, 2010.

38. Cha HJ, Bae SK, Lee HY, et al: Anti-invasive activity of ursolic acid correlates with the reduced expression of matrix metalloproteinase-9 (MMP-9) in HT1080 human fibrosarcoma cells. Cancer Res 56: 2281-2284, 1996.

39. Yang L, Liu X, Lu Z, et al: Ursolic acid induces doxorubicin-resistant HepG2 cell death via the release of apoptosis-inducing factor. Cancer Lett 298: 128-138, 2010.

40. Heaney AP, Fernando M, Yong WH and Melmed S: Functional PPAR-gamma receptor is a novel therapeutic target for ACTH-secreting pituitary adenomas. Nat Med 8: 1281-1287, 2002.

41. Schaaf C, Shan B, Buchfelder M, et al: Curcumin acts as anti-tumorigenic and hormone-suppressive agent in murine and human pituitary tumour cells in vitro and in vivo. Endocr Relat Cancer 16: 1339-1350, 2009.

42. Green DR: Apoptotic pathways: paper wraps stone blunts scissors. Cell 102: 1-4, 2000.

43. Nagata S: Apoptosis by death factor. Cell 88: 355-365, 1997.

44. Kroemer G and Reed JC: Mitochondrial control of cell death. Nat Med 6: 513-519, 2000.

45. Kuwana T and Newmeyer DD: Bcl-2-family proteins and the role of mitochondria in apoptosis. Curr Opin Cell Biol 15: 691-699, 2003.

46. Achiwa Y, Hasegawa K and Udagawa Y: Regulation of the phosphatidylinositol 3-kinase-Akt and the mitogen-activated protein kinase pathways by ursolic acid in human endometrial cancer cells. Biosci Biotechnol Biochem 71: 31-37, 2007.

47. Zhang YX, Kong CZ, Wang LH, et al: Ursolic acid overcomes Bcl-2-mediated resistance to apoptosis in prostate cancer cells involving activation of $\mathrm{JNK}$-induced $\mathrm{Bcl}-2$ phosphorylation and degradation. J Cell Biochem 109: 764-773, 2010.

48. Cheng EH, Wei MC, Weiler S, et al: BCL-2, BCL-X(L) sequester $\mathrm{BH} 3$ domain-only molecules preventing BAX- and BAK-mediated mitochondrial apoptosis. Mol Cell 8: 705-711, 2001. 